\title{
artigo
}

Santos, A.O.; Araújo, I.O.; Bitencourt, J.M.M.; Santos, V.H.D.S.; Moura, M.M.; Humia, B.V.; Santos, L.X.C.; Oliveira, R.I.S.;

Incidência dos agrotóxicos no organismo humano: revisão de literatura

\section{Incidência dos agrotóxicos no organismo humano: revisão de literatura}

\author{
Incidence of pesticides on human organism: literature review
}

Incidencia de plaguicidas en el organismo humano: revisión de la literatura

\begin{abstract}
RESUMO
Objetivo: Evidenciar os impactos do uso de agrotóxicos no organismo humano que ocasionam diversos problemas de saúde e, em alguns casos, levam a fatalidade, trazendo métodos utilizados para combater a intoxicação. Método: Realizou-se o estudo por revisão bibliográfica integrativa, selecionando artigos pelos bancos de dados SciELO, PubMed, MEDLINE e a plataforma BVS, nos quais foram retiradas publicações de 2016 a 2021. Resultados: A temática exposta mostrou-se importante podendo assim ser utilizado com o intuito de proporcionar conhecimento, principalmente aos trabalhadores rurais que fazem o manuseio dos agrotóxicos. Conclusão: Neste estudo, foi observado grande importância na temática em questão, uma vez que o mesmo possibilitou observar os malefícios causados na vida dos agricultores que são os mais afetados, por terem um contato direto com os agroquimicos, e de toda a população que consome alimentos contaminados em excesso, trazendo novos conhecimentos aos profissionais de saúde, em especial aos biomédicos.
\end{abstract}

DESCRITORES: Agrotóxicos; Trabalhadores rurais; Exposição ocupacional; Intoxicação; Biomédico.

\section{ABSTRACT}

Objective: To show the impacts of the use of pesticides on the human body that cause various health problems and, in some cases, lead to fatalities, bringing methods used to combat intoxication. Method: The study was carried out by an integrative bibliographic review, selecting articles from the SciELO, PubMed, MEDLINE databases and the BVS platform, in publications from 2016 to 2021. Results: The exposed theme proved to be important and can be used to provide knowledge, especially to rural workers who handle pesticides. Conclusion: In this study, a great importance was observed in the subject in question, as it made it possible to observe the harm caused in the lives of farmers who are most affected by having direct contact with agrochemicals, and of the entire population that consumes contaminated food in excess, bringing new knowledge to health professionals, in particular to biomedical scientis.

DESCRIPTORS: Pesticides; Rural workers; Occupational exposure; Intoxication; Biomedic.

\section{RESUMEN}

Objetivo: Mostrar los impactos del uso de plaguicidas en el cuerpo humano, que ocasionan diversos problemas de salud y, en algunos casos, conducen a fatalidades, con la intención de acercar los métodos utilizados para combatir las intoxicaciones. Método: El estudio se realizó mediante una revisión bibliográfica integradora, seleccionando artículos de las bases de datos SciELO, PubMed, MEDLINE y la plataforma BVS, en publicaciones de 2016 a 2021. Resultados: El tema expuesto demostró ser importante y por lo tanto puede utilizarse para brindar conocimientos, especialmente a los trabajadores rurales que manipulan plaguicidas. Conclusión: En este estudio se observó gran importancia en el tema en cuestión, ya que permitió observar el daño causado en la vida de los agricultores más afectados, al tener contacto directo con agroquímicos, y de toda la población que consume alimentos contaminados en exceso, aportando nuevos conocimientos a los profesionales de la salud, especialmente a los biomédicos.

DESCRIPTORES: Plaguicidas; Trabajadores rurales; Exposición ocupacional; Intoxicación; Biomédica.

RECEBIDO EM: 09/06/2021 APROVADO EM: 16/06/2021

\section{Amannda Oliveira Santos}

Graduando(a) em Biomedicina, Centro Universitário Estácio de Sergipe, Aracaju, Sergipe, Brasil.

ORCID: 0000-0003-3287-7272 
Isadora Oliveira de Araújo

Graduando(a) em Biomedicina, Centro Universitário Estácio de Sergipe, Aracaju, Sergipe, Brasil.

ORCID: 0000-0001-5920-0750

\section{João Marcos Mendes Bitencourt}

Graduando(a) em Biomedicina, Centro Universitário Estácio de Sergipe, Aracaju, Sergipe, Brasil.

ORCID: 0000-0002-0199-716X

\section{Victor Hugo Dias Silva Santos}

Graduando em Enfermagem, Centro Universitário Estácio de Sergipe, Aracaju, Sergipe, Brasil.

ORCID: 0000-0001-6074-2047

\section{Mayra Morgana Martins de Moura}

Graduada em Biomedicina, Centro Universitário Lusíada (UNILUS), Santos, São Paulo, Brasil.

ORCID: 0000-0002-4773-1834

\section{Bruno Vieira Humia}

Professor no Centro Universitário Estácio de Sergipe, Graduado em Biomedicina pela Escola Bahiana de Medicina e Saúde

Pública, Mestre e Doutor em Biotecnologia Industrial pela Universidade Tiradentes, Aracaju, Sergipe, Brasil.

ORCID: 0000-0002-4349-6064

\section{Lorena Xavier Conceição Santos}

Professora no Centro Universitário Estácio de Sergipe, Graduada em Ciências Biológicas e Mestre em Saúde e Ambiente pela Universidade Tiradentes, Doutora em Desenvolvimento e Meio Ambiente pela Universidade Federal de Sergipe, Aracaju, Sergipe, Brasil.

ORCID: 0000-0001-5995-2442

\section{Raphaella Ingrid Santana Oliveira}

Orientadora/Professora no Centro Universitário Estácio de Sergipe, Graduada em Biomedicina e Mestre em Biotecnologia Industrial pela Universidade Tiradentes, Aracaju, Sergipe, Brasil.

ORCID: 0000-0002-7027-9049

\section{INTRODUÇÃO}

0 $s$ agrotóxicos são definidos como qualquer substância ou mistura de substâncias químicas ou biológicas empregadas com o objetivo de repelir, destruir, controlar pragas ou regular o crescimento da planta ${ }^{(1)}$. Entretanto, também é possível notar que tais substâncias podem proporcionar contaminação dos rios e lagos, pois os pesticidas depositados nas lavouras penetram no solo, escorrem chegando até os rios e acabam sendo um agente causador de poluição( ${ }^{(2)}$.

Em tese, todas as pessoas estão expostas aos malefícios dos agrotóxicos, porém, existem as mais vulneráveis, como idosos, crianças, gestantes e as pessoas que moram nas proximidades rurais onde é pulverizado e, principalmente, aqueles que lidam diretamente com os produtos, como os trabalhadores rurais e familiares, por haver contaminação coletiva ${ }^{(3)}$.
A exposição aos agrotóxicos ocorre, principalmente, no setor agropecuário, nas empresas desinsetizadoras, durante o transporte, na comercialização e na produção de agrotóxicos ${ }^{(4)}$. Além da exposição ocupacional, destaca-se a contaminação alimentar e ambiental, que coloca em risco a saúde de outros grupos populacionais, como as famílias dos agricultores e a população em geral que se alimenta do que é produzido no campo ${ }^{(5)}$.

Os pesticidas são usados em grande escala por vários setores produtivos, como tratamento de madeira para a construção, armazenamento de grãos e sementes, produção de flores e combate às endemias $\mathrm{e}$ epidemias $^{(6)}$. A classificação dos mesmos, de acordo com o grupo químico, inclui, principalmente, os organoclorados, clorofosforados, piretroides, organofosforados e carbamatos ${ }^{(7)}$. Assim, o impacto negativo para a saúde dos consumidores e dos trabalhadores que manejam frequen- temente esses produtos tóxicos dependem de uma série de fatores do tipo (toxicidade) do agrotóxico utilizado, da forma de aplicação dos produtos; dos mecanismos de vigilância da saúde, da falta do não uso ou do uso inadequado dos equipamentos de proteção individuais e coletivos (EPI's e EPC's); do modo de produção agrícola, dentre outros ${ }^{(8)}$. Somado a esses fatores estão o baixo nível de escolaridade e as precárias condições socioeconômicas e culturais de muitos trabalhadores rurais. Estes, não raros, adoecem inúmeras vezes, porém, estes eventos não são contabilizados ou não relacionados com os produtos químicos da agroindústria ${ }^{(9)}$.

Os agroquímicos deixam um rastro deletério, desde a sua produção até a sua utilização, passando por aqueles que transportam estes produtos e os que consomem os alimentos contaminados nas regiões em que este método de controle de pragas seja o escolhido ${ }^{(3)}$. Como os agrotóxicos 
são substâncias que possuem como principal finalidade a proteção dos produtos agrícolas contra a ação de seres vivos nocivos e, por muitas vezes, não são aplicados utilizando os equipamentos de proteção adequados, acabam gerando riscos à saúde humana ${ }^{(10)}$.

Desta maneira, torna-se pertinente o melhor estudo sobre o tema a fim de explanar sobre os malefícios que os agrotóxicos podem causar na saúde humana, trazendo caminhos eficazes e satisfatórios para a diminuição da utilização e consumo dos agrotóxicos, mostrando que pode ser feito um modelo de agricultura mais sustentável, garantindo uma alimentação mais saudável, melhora na qualidade de vida e, podendo assim, aumentar a longevidade das pessoas, garantindo um solo mais saudável para o plantio. Assim, o trabalho teve como objetivo evidenciar os impactos do uso de agrotóxicos no organismo humano, conhecer as consequências fisiopatológicas da exposição humana a resíduos de agrotóxicos, trazendo métodos utilizados para combater a intoxicação.

\section{MÉTODOS}

O estudo baseou-se em uma pesquisa bibliográfica do tipo revisão integrativa, ou seja, Baseada em Evidências (PBE). A revisão integrativa consiste no cumprimento das seguintes etapas: identificação do tema e sele- ção da questão de pesquisa; estabelecimento de elegibilidade; identificação dos estudos nas bases científicas; avaliação dos estudos selecionados e análise crítica; classificação dos estudos; avaliação e interpretação dos resultados e apresentação dos dados na estrutura do estudo apresentado ${ }^{(11)}$.

Ao comensurar a abordagem referente à incidência dos agrotóxicos na saúde humana, elaboramos as seguintes questões norteadoras: Quais os métodos para diminuir a agressão dos agrotóxicos nos alimentos, para que seja menos prejudicial à saúde dos trabalhadores agrícolas e consumidores dos produtos? De qual forma o biomédico pode atuar frente a esse problema?

O levantamento bibliográfico desse estudo foi realizado no período de fevereiro a abril de 2021. Na fundamentação descrita para a pesquisa, realizou-se pela revisão bibliográfica de artigos internacionais e nacionais, em síntese, selecionados pelo: Scientific Eletronic Library Online (SciELO), Biblioteca Virtual da Saúde (BVS), Biblioteca Nacional de Medicina (PubMed) e Medical Literature Analysis and Retrieval System Online (MEDLINE).

Para o critério de inclusão, foram incluídos artigos publicados nos últimos 5 anos (2016 a 2020), disponíveis nas plataformas de busca, no idioma inglês e português, envolvendo quatro palavras com grandes significados para o assunto: "Agrotóxicos", "Trabalhadores Rurais", "Exposição Ocu-

GRÁFICO 1 - Distribuição dos anos de publicação dos artigos (2016-2021).

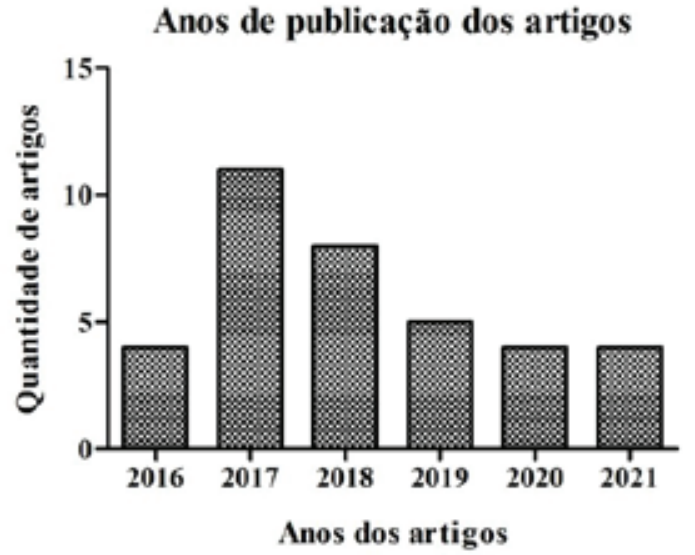

Fonte: Autoria própria (2021). pacional", "Intoxicação" e "Biomédico". O critério de exclusão foi respaldado nos artigos que não apresentaram uma síntese direta do assunto e alguns foram repetidos, desse modo, não atendeu as bases legais e objetivas da pesquisa.

A avaliação dos resultados ocorreu através de gráficos executados no programa GraphPad PRISM ${ }^{\circ}$ versão 5.00. e tabela com as seguintes informações: Autor, Revista/Ano, Título, Método e Resultado. Dessa forma foi possível a observação, classificação e discussão dos dados obtidos.

\section{RESULTADOS}

A pesquisa permitiu encontrar 2.856 artigos utilizando as palavras selecionadas na metodologia, mas somente 36 artigos foram utilizados neste estudo, dentro do parâmetro de pesquisa estabelecido (20162021). Após análise e avaliação dos estudos selecionados, foram excluídos 2.820 artigos. Diante disso, o Gráfico 1 demonstra a distribuição dos anos de publicação dos artigos, em relação ao quantitativo de publicação. De acordo com ${ }^{(12)}$, a série histórica dos casos notificados de intoxicações por agrotóxicos no Sistema de Informações de Agravos de Notificação (SINAN), entre 2007 e 2015, foi notificada no Brasil em um total de 84.206 casos e, com isso, possibilitou o surgimento de novos estudos publicados, principalmente no ano de 2017 onde teve mais relevância neste artigo. Após 2017, os anos com mais relevância neste estudo foram 2018, 2019, 2020, 2016, nesta ordem, sendo o menor quantitativo em 2021, talvez por ainda estarmos no ano vigente.

O Gráfico 2 demonstra a quantidade de artigos por base de dados, destacando a maioria dos artigos na plataforma SciELO, seguida das plataformas BVS, PubMed e MEDLINE, de acordo com os descritores utilizados para a pesquisa. Segundo Spinak(13), a plataforma SciELO compreende mais de 1.200 periódicos ativos, de acesso aberto e com texto completo de 17 países, também possui mais de 900.000 artigos e o SciELO Livros, disponibilizando 1.300 livros. Diante disso, o resultado sugere que o SciELO obteve mais relevância 
neste estudo por ser mais abrangente e de mais fácil acesso.

\section{DISCUSSÃO}

Segundo ${ }^{(14,15)}$, a área de plantação no Brasil proporcionou que o país fosse o maior consumidor de agrotóxicos no mundo, pois a imposição da Política da Revolução Verde, dos cultivos transgênicos, o aumento de "pragas" nas lavouras, de créditos agrícolas subsidiados e isenção de tributos fiscais, são fatores que contribuíram para o aumento no consumo de agrotóxicos na agropecuária. De acordo com $^{(16)}$, o crescimento populacional em conjunto com a escassez dos alimentos, fez com que a utilização dos agrotóxicos aumentasse cada vez mais, com o intuito de obter um método de produção mais acelerado e, com isso, também obter bons resultados na produção.

De acordo com $^{(17)}$, conforme citado por $^{(18)}$, esse grande consumo, tem causado impacto negativo à saúde humana e ao meio ambiente causado pela exposição aguda e crônica aos agrotóxicos, que já é bem conhecida pela literatura e, apesar disso, contraditoriamente, o Brasil consome imensas quantidades destes venenos.

Segundo ${ }^{(19,20)}$, o uso de compostos químicos sintéticos para controle de pragas possui pontos negativos, pois afetam a flora, fauna, solo, animais, microrganismos, trabalhadores rurais e aos integrantes do

\section{GRÁFICO 2 - Distribuição do quantitativo de artigos utilizados por base de dados.}

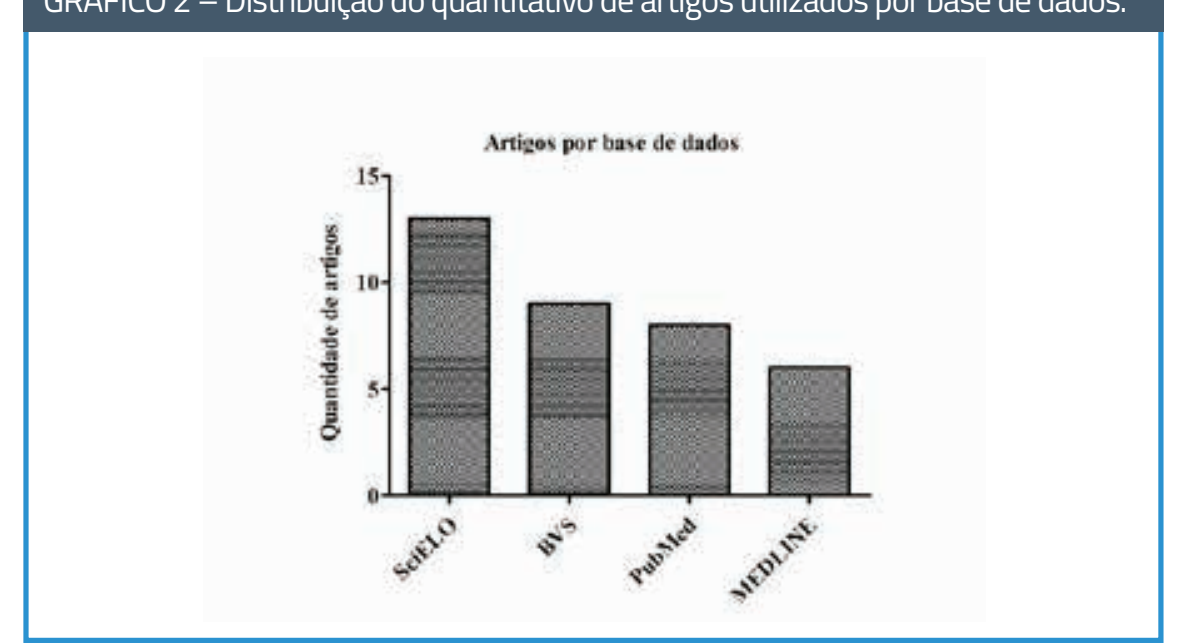

mercado consumidor. De acordo com $^{(21,22)}$, o uso desses compostos vem causando efeitos que não refletem somente em benefícios sociais, mas sim em problemas ambientais e de saúde pública, sendo responsáveis por complexas contaminações químicas, especialmente, no meio rural.

Vale ressaltar que a poluição química de natureza orgânica ou inorgânica ocasiona um dos problemas sérios ao meio ambiente, sendo decorrente da introdução de pesticidas, fertilizantes, produtos químicos em ge$\mathrm{ral}^{(23,24)}$. Também, utilização de fertilizantes e agrotóxicos nas lavouras podem causar a contaminação do solo, uma vez que muitos fertilizantes possuem metais pesados em sua composição contribuindo para o conteúdo tóxico do local(25,24).

Algumas questões têm sido levantadas a respeito da possibilidade de um risco aumentado de contaminação microbiológica e parasitária nos alimentos produzidos, em virtude principalmente do tipo de adubação, proveniente de esterco de animal e com a proibição do uso de agrotóxicos, tornando assim o alimento inapropriado para o consumo humano ${ }^{(16) .}$

Em um estudo feito por ${ }^{(26,27)}$, ressalta-se que $52 \%$ não utilizam roupas impermeáveis e $44 \%$ não utilizam luvas. A utilização de EPI's, como máscara com filtro, macacão com mangas longas, óculos ou viseira, luvas e botas, apesar de desconfortáveis, deve ser considerado como tecnologia de

Fonte: Autoria própria (2021). proteção disponível e indispensável dentro de uma abordagem dos problemas ocupacionais, no caso dos agroquímicos, onde os EPI's são projetados para garantir proteção contra os agentes químicos externos, ou seja, que esses agentes não entrem em contato com o organismo ${ }^{(22)}$.

Outrossim $^{(28,29)}$, também realizaram um estudo onde encontraram como principais sintomas apresentados pelos agricultores, após a exposição, tontura (presente em $22 \%$ dos trabalhadores entrevistados), dor de cabeça (16\%), visão turva (11\%), náusea (11\%), diarreia (8\%), suor (8\%) e cólica abdominal (8\%). Então, diante desses estudos dispostos na literatura, é possível caracterizar as intoxicações por agrotóxicos reportadas a um centro de assistência toxicológica, por meio de análise retrospectiva de fichas epidemiológicas de ocorrência toxicológica, com diagnóstico médico de intoxicação aguda ou crônica por agrotóxicos ${ }^{(30)}$.

A análise de ocorrência é feita através do Sistema de Informações de Agravos de Notificação (SINAN) após atendimento médico, com o objetivo de registrar o processamento dos dados sobre agravos de notificação em todo o território nacional, fornecendo informações para análise do perfil dos indivíduos com exposição e/ou intoxicação por agrotóxicos no Brasil ${ }^{(31)}$. Para isso, torna-se pertinente que a população seja instruída e que programas de capacitação profissional de assistência a esses pacientes ocorram de forma contínua para que seja viável o acompanhamento retrospectivo desses pacientes acometidos, bem como o levantamento de casos no país ${ }^{(32)}$.

Em um estudo de caso, realizado por $^{(33,34)}$ após assinatura do termo de consentimento, 62 participantes responderam a um questionário sobre o processo e as relações de trabalho na fumicultura, inserção na produção e no consumo, bem como problemas de saúde percebidos como advindos dessa atividade. Desse total, 46 pessoas participaram da segunda fase, composta por anamnese e exame físico, aplicação do Self-Reporting Questionnaire (SRQ-20), validado no País em usuários de serviços de saúde da atenção primária, além de coleta de sangue para realização de exames 


\section{artigo}

Santos, A.O.; Araújo, I.O.; Bitencourt, J.M.M.; Santos, V.H.D.S.; Moura, M.M.; Humia, B.V.; Santos, L.X.C.; Oliveira, R.I.S.

Incidência dos agrotóxicos no organismo humano: revisão de literatura

de: colinesterase eritrocitária e plasmática, hemograma com contagem de reticulócitos, ureia, creatinina, proteínas totais e frações, bilirrubinas totais e frações, fosfatase alcalina, Transaminase Aspartato Aminotransferase (AST), Transaminase Alanina Aminotransferase (ALT), Gama Glutamil Transferase (GAMA-GT), hormônio tireoestimulante (TSH), glicemia de jejum, colesterol total e frações, triglicerídeos, Estudo Laboratorial de Doenças Venéreas (VRDL) ${ }^{(33)}$. Esses exames foram realizados para definir a intoxicação/envenenamento de acordo com a exposição ao agente químico que resultou em alterações clínicas e/ ou laboratoriais compatíveis com o quadro de exposição tóxica, podendo ser agudas (exposição única por até 24 horas, ou repetida por até 15 dias), subcrônicas (repetidas entre 15 dias e três meses) e crônicas (acima de três meses) $)^{(35)}$.

Desta forma, percebe-se que a avaliação de pacientes que tem histórico advindo de agrotóxicos também se faz através de exames clínicos e laboratoriais. Assim, o modelo biomédico de saúde com o seu foco na etiologia, diagnóstico e tratamento das doenças tem dado uma importante contribuição ao desenvolvimento da assistência médica. No entanto, essa abordagem reducionista, em geral, não leva em consi- deração fatores que influenciam a saúde, como o ambiente físico e social onde os problemas de saúde ocorrem ${ }^{(36)}$.

A Política Nacional de Agroecologia de Produção Orgânica (PNAPO) é um modelo de agricultura sustentável que se aplica aos conceitos e princípios ecológicos para o estudo e manejo dos sistemas agrícolas, gerando base científica para desenvolver uma agricultura mais sustentável, fundamentada na valorização da diversidade cultural e biológica ${ }^{(37)}$. Essa prática busca conservar e resgatar as variedades crioulas e o conhecimento tradicional das populações locais, diferentemente da forma de difusão empregada na agricultura convencional, promovida por meio de pacotes tecnológicos, gerando conhecimento por meio da socialização e troca de saberes entre comunidades agrícolas e urbanas ${ }^{(38)}$.

Desta forma, é possível compreender que os agrotóxicos estão presentes na população como forma de aumento da produção e renda dos agricultores e com o incentivo à agroindústria de exportação. Entretanto, com o decorrer do tempo, vem causando danos muitas vezes irreversíveis a população, principalmente aos produtores por ter contato direto ${ }^{(39)}$. Assim, vimos a importância do monitoramento visando a redução e controle dos agrotóxicos na produção dos alimentos, fazendo com que seja perceptível e de suma importância também, a utilização de (EPI's) durante a aplicação dos venenos nas lavouras, evitando o contato direto aos agroquímicos. O risco de contaminação e agravos à exposição são notórios, desta forma, é possível compreender que os agrotóxicos estão causando danos à saúde da população e do meio ambiente ${ }^{(40)}$.

\section{CONCLUSÃO}

O trabalho tratou sobre os malefícios que os agrotóxicos podem causar à saúde humana, apresentando caminhos satisfatórios para o uso adequado e a diminuição da utilização dos agrotóxicos, visando garantir uma alimentação mais saudável, possibilitando assim, a melhoria na qualidade de vida das pessoas. Também ressaltou a importância do biomédico para o diagnóstico das intoxicações, realizando os exames laboratoriais após atendimento médico. Palestras e anúncios também mostram à população os perigos da exposição a essas substâncias e a importância de EPI's adequados, tendo esses como um dos principais métodos que influenciam a diminuição da incidência de contaminação com os agrotóxicos. Com isso, espera-se que este estudo favoreça uma discussão futura para novas pesquisas.

\section{REFERÊNCIAS}

1. Nogueira FAM. Exposição a agrotóxicos e agravos à saúde em trabalhadores agrícolas: o que revela a literatura? Rev Bras Saude Ocup 2020; 45(36).

2. Lopes CVA, Albuquerque GSC. Agrotóxicos e seus impactos na saúde humana e ambiental: uma revisão sistemática. Saúde debate 2018; 42(117).

3. Agrotóxicos e as repercussões na saúde dos trabalhadores rurais: revisão de literatura [Mestrado]. Pombal/PB: Universidade Federal de Campina Grande - UFCG; 2018.

4. Silva JM, Silva EN, Faria HP, Pinheiro TMM. Agrotóxico e trabalho: uma combinação perigosa para a saúde do trabalhador rural. Ciência \& saúde coletiva 2005; 10(4).

5. Almeida MD, Cavendish TA, Bueno PC, Ervilha IC, Gregório LS, Kanashiro NBO, et al. A flexibilização da legislação brasileira de agrotóxicos e os riscos à saúde humana: análise do Projeto de Lei no 3.200/2015. Cadernos de Saúde Pública 2017; 33(7).

6. Ministério da Saúde (BR). Documento Orientador para a Im- plementação da Vigilância em Saúde de Populações Expostas a Agrotóxicos. Brasília/DF: Ministério da Saúde; 2013.

7. Santana CM, Costa AR, Nunes RMP, Nunes NMF, Peron AP, Cavalcante AACM et al. Exposição ocupacional de trabalhadores rurais a agrotóxicos. Cadernos Saúde Coletiva 2016; 24(3).

8. Carneiro FF, Rigotto RM, Augusto LGS, Pignati W, Rizzolo A, Alexandre VP, et al. Dossiê ABRASCO: um alerta sobre os impactos dos agrotóxicos na saúde, $1^{\text {a }}$ parte, Rio de Janeiro; 2012.

9. Borges LD. Agrotóxicos e seus efeitos na saúde pública: um panorama evolutivo. Caderno de pós-graduação em análise ambiental e desenvolvimento sustentável: legislação ambiental. Centro Universitário de Brasília - UniCEUB; 2016.

10. Gomes ACS, Moraes LGS, Moraes CRS. O uso de agrotóxicos e a saúde do trabalhador rural no Brasil. ARIGÓ - Revista do Grupo PET e Acadêmicos de Geografia da Ufac 2018; 1(1).

11. Ercole, FF, Melo SL, Alcoforado CLGC. Revisão Integrativa versus Revisão Sistemática. Revista Mineira de Enfermagem 


\section{REFERÊNCIAS}

2014; 18(1).

12. Ministério da Saúde (BR). Relatório Nacional de Vigilância em Saúde de Populações Expostas a Agrotóxicos. Brasília/DF: Ministério da Saúde; 2018.

13. Spinak E. Integração de bases de dados nacionais acadêmicas na Europa. SciELO em Perspectiva 2021.

14. Carneiro FF, Augusto LGS, Rigotto RM, Friedrich $K$, Búrigo AC. Dossiê ABRASCO: um alerta sobre os impactos dos agrotóxicos na saúde. Rio de Janeiro: EPSJV; São Paulo: Expressão Popular; 2015.

15. Pignati WA, Lima FANS, Lara SS, Correa MLM, Barbosa JR, Leão LHC, et al. Distribuição espacial do uso de agrotóxicos no Brasil: uma ferramenta para a Vigilância em Saúde. Ciência \& Saúde Coletiva 2017; 22(10).

16. Fidelis RA, Gonçalves AV, Santos FAL. Contaminação, por agrotóxicos, bactérias, protozoários, helmintos e artrópodes, nas hortaliças orgânicas e convencionais comumente relatados na literatura. [Resumo]. Centro Universitário de Várzea Grande UNIVAG 2017.

17. Rigotto RM, Vasconcelos DP, Rocha MM. Uso de agrotóxicos no Brasil e problemas para a saúde pública. Caderno de Saúde Pública 2014; 30(7).

18. Lopes CVA, Albuquerque GSC. Desafios e avanços no controle de resíduos de agrotóxicos no Brasil: 15 anos do Programa de Análise de Resíduos de Agrotóxicos em Alimentos. Caderno de Saúde Pública 2021; 37(2).

19. Martins, MAR. O trabalhador rural e os agrotóxicos. Revista Direito, Trabalho e Política Social 2015; 1(1).

20. Coutinho, KA. Intoxicações relacionadas a exposição por agrotóxicos [Monografia]. Brasília/DF: Centro Universitário se Brasília - UniCEUB; 2017.

21. Vieira MG, Steinke G, Arias JLO, Primel EG, Cabrera LCC. Avaliação da Contaminação por Agrotóxicos em Mananciais de Municípios da Região Sudoeste do Paraná. Revista Virtual de Química 2017; 9(5).

22. Martins DE. Impactos ambientais da utilização de agrotóxicos: percepção dos trabalhadores rurais e adesão a métodos alternativos [Mestrado] Chapecó/SC: Universidade Federal da Fronteira Sul; 2019.

23. Aguiar MRMP, Novaes AC, Guarino AWS. Remoção de metais pesados de efluentes industriais por aluminossilicatos. Química Nova 2015; 25(6b):1145-54.

24. Oliveira RIS, Araújo SS, Lisboa CLS, Resende AF, Santos TS, Alves LL, Alves LL, Lima Silva W. Análise comparativa de elementos físico-químicos e contaminantes em corpo d'água do Riacho Grilo no estado de Sergipe. Saúde Coletiva 2020; 10(59).

25. Silva ES, Jesus RS. Caracterização Geoambientais do Rio Machado na área do bairro Matinha em Lagarto/SE. [Monografia]. Lagarto/SE: Universidade Federal de Sergipe; 2012.

26. Pacheco FP, Vasconcelos HL. Saúde e segurança do trabalho: agentes químicos e equipamentos de proteção individual utilizados no tratamento e manuseio de sementes tratadas. Revista Varia Scientia Agrárias 2013; 3(2).

27. Silva MBC. Avaliação da exposição a agrotóxicos em pacientes com leucemia [TCC]. Santa Cruz do Sul/RS: Universidade De Santa Cruz Do Sul; 2020.

28. Santos LDC, Oliveira MFF, Rodrigues MA, Barbosa PM, Sercundes SP. Investigação sobre o manejo e aplicação de agrotóxicos pelos agricultores da fazenda boa vista, do município de Goioerê - PR e do vilarejo Água Santos Antônio, do município de Janiópolis - PR, 2000. Arquivos da Apadec 2001; 5(1).

29. Taveira BLS, Albuquerque GSC. Análise das notificações de intoxicações agudas, por agrotóxicos, em 38 municípios do estado do Paraná. Saúde Debate 2018; 42(4).

30. Hungaro AA, Correia LM, Silvino MCS, Rocha SM, Martins BF, Oliveira MLF. Intoxicações por agrotóxicos: registros de um serviço sentinela de assistência toxicológica. Ciência, cuidado e saúde 2015; 14(3).

31. Malaspina FG, ZiniLise ML, Bueno PC. Perfil epidemiológico das intoxicações por agrotóxicos no Brasil, no período de 1995 a 2010. Caderno de Saúde Coletiva 2011; 19(4).

32. Ambrosini MB, Witt RR. As intoxicações por agrotóxicos no meio rural e a atuação do enfermeiro. Revista Gaúcha de Enfermagem 2000; 21(1).

33. Mari JJ, Williams P. A validity study of a psychiatric screening questionnaire (SRQ-20) in primary care in the city of São Paulo. British Journal of Psychiatry 1986.

34. Murakami Y, Pinto NF, Albuquerque GSC, Perna PO, Lacerda A. Intoxicação crônica por agrotóxicos em fumicultores. Saúde Debate 2017; 41(113).

35. Germano LC, Alonzo HGA. Estudo descritivo dos atendimentos hospitalares por eventos toxicológicos em um município do estado de São Paulo, 2012. Epidemiologia e Serviços de Saúde 2017; 26(3).

36. Souza EM, Grundy E. Promoção da saúde, epidemiologia social e capital social: inter-relações e perspectivas para a saúde pública. Caderno de Saúde Pública 2004; 20(5).

37. Moura IF. Agroecologia na agenda governamental brasileira: trajetórias no período 2003-2014 [Tese]. Rio de Janeiro: Universidade Federal Rural Do Rio De Janeiro; 2016.

38. Sambuichi RHR, Oliveira MAC, Silva APM, Luedemann G. A sustentabilidade ambiental da agropecuária brasileira: impactos, políticas públicas e desafios. Rio de Janeiro: IPEA - Instituto de Pesquisa Econômica Aplicada; 2012.

39. Miranda AC, Moreira JC, Carvalho R, Peres F. Neoliberalismo, uso de agrotóxicos e a crise da soberania alimentar no Brasil. Ciência \& Saúde Coletiva 2007; 12(1).

40. Silva JB, Xavier DS, Barboza MCN, Amestoy SC, Trindade LL, Silva JRS. Fumicultores da zona rural de Pelotas (RS), no Brasil: exposição ocupacional e a utilização de equipamentos de proteção individual (EPI). Saúde em Debate 2013; 37(97). 